\title{
Thermal diffusion of solitons on anharmonic chains with long-range coupling
}

\author{
C. Brunhube1* and F.G. Mertens \\ Physikalisches Institut, Universität Bayreuth, D-95440 Bayreuth, Germany \\ Y. Gaididei \\ Bogolyubov Institute for Theoretical Physics, 03143 Kiev, Ukraine
}

\begin{abstract}
We extend our studies of thermal diffusion of non-topological solitons to anharmonic FPU-type chains with additional long-range couplings. The observed superdiffusive behavior in the case of nearest neighbor interaction (NNI) turns out to be the dominating mechanism for the soliton diffusion on chains with long-range interactions (LRI). Using a collective variable technique in the framework of a variational analysis for the continuum approximation of the chain, we derive a set of stochastic integro-differential equations for the collective variables (CV) soliton position and the inverse soliton width. This set can be reduced to a statistically equivalent set of Langevin-type equations for the CV, which shares the same Fokker-Planck equation. The solution of the Langevin set and the Langevin dynamics simulations of the discrete system agree well and demonstrate that the variance of the soliton increases stronger than linearly with time (superdiffusion). This result for the soliton diffusion on anharmonic chains with long-range interactions reinforces the conjecture that superdiffusion is a generic feature of non-topological solitons.
\end{abstract}

\section{INTRODUCTION}

Solitons appear in various different fields of physics such as solid state physics, quantum theory, nonlinear optics, fluid dynamics, biophysics or even cosmology 1, 2] . The numerical experiment done by Fermi, Pasta and Ulam in 1955, investigating the thermalization of a onedimensional chain with anharmonic interaction potential, was certainly one of the key events in nonlinear science 3]. Hereafter, FPU chains, despite of their simplicity, served as frequently used models for analyzing the properties of microscopic structures, especially in computer simulations. More than fifty years after the above mentioned experiment by Fermi, Pasta and Ulam, complete understanding of this putatively simple system, which still reveals new astonishing phenomena [4], is yet to be reached. The thermalization of the FPU system, the heat conduction in FPU systems or the properties of lattice solitons and discrete breathers are still tasking many physicists (see for example CHAOS Volume 15: focus issue: THE "FERMI-PASTA-ULAM" PROBLEM-THE FIRST 50 YEARS).

Physicists usually use simplified models to describe and explain the most important features of more complex systems. An example is the "invasion" of physicists into biology, where they aimed to explain some of the most interesting processes in living cells such as the DNA dynamics (DNA melting, DNA transcription) or the functionality of proteins as molecular motors, and the storage and transport of energy released by the hydrolysis of adenosine-triphosphate (ATP) [5, [6, 7, 8, 9, 10]. Oversimplified models, which were formerly used to predict freely moving solitons along DNA or protein $\alpha$ helices may be too rough to explain the dynamics of

*Electronic address: Christian.Brunhuber@uni-bayreuth.de biomolecules. But, for example, taking the role of discreteness, thermal perturbations and the underlying geometry of the system into account, it is possiblee to obtain good agreements with the results of experiments, which today work at the scale of single molecules.

The Davydov model 10, 11] for energy transport and storage in proteins has attracted much attention in the past. Davydov proposed a self-trapping of vibrational energy of the $\mathrm{C}=\mathrm{O}$ vibration, similar to the concept of polarons in solid-state physics. Recently, a direct observation of self-trapped states in pump-probe experiments of a model protein $(\mathrm{ACN})$, showed that the self-trapping could describe some important features of proteins (although the lifetime of the excitation in isolated molecules is shorter than expected by Davydov) [12]. In a different approach by Yomosa [13], a molecular mechanism of muscle contraction was introduced, which assumes that the H-bonding interaction of the peptide groups (which stabilize the helix structure) can be modeled by a FPUtype lattice. The energy transport along the helix was described in the continuum approximation by use of the stability of the Korteweg-de Vries (KdV) or the Toda soliton [14, 15, 16]. Although the continuum approximation and the disregard of the helical structure seems to be bold to model real processes, the concept of lattice solitons in protein backbones was further refined. It was numerically demonstrated that protein backbones with a more realistic structure, support stable non-topological solitons [17]. Recently, the concept of solitons along hydrogen bonds in $\alpha$ helices was applied to realistic models for proteins in order to explain Raman measurements, which identify excitations in proteins, which can not stem from vibrational modes in the molecule and are too long-lived to result from large-scale vibrations of the protein [18, 19]. The three-dimensional model of the $\alpha$ helix consists of three chains with hydrogen bonds (secondary structure), which are connected with peptide bonds (primary structure), modeled by Lennard-Jones or Toda potentials. It 
was demonstrated that a perturbation in one of the three $\mathrm{H}$-bond lines can create a triple-soliton in the three hydrogen bonds, which travels (phase-locked by the peptide bonds) along the chain and would present a long-lived energy source for the spectroscopically observed phonons at energies of about $\sim 100 \mathrm{~cm}^{-1}$.

Often, the dynamics of more realistic models can only be examined or dealt with in computer simulations. Yet, sometimes simplified models, such as the FPU-chain, are able to provide proper understanding of these models. Therefore, it is important to clarify the effect of extensions on systems such as FPU-chain and if possible, apply analytical calculations. In the case of biomolecules, especially damping and thermal fluctuations appear as natural perturbations. The influence of the thermal fluctuations on solitons on FPU chains (with cubic or quartic anharmonicity) has recently been investigated 20, 21, 22, 23]. It was demonstrated that both types of non-topological solitons, pulse solitons and envelope solitons, show a position variance growing stronger than linearly in time (superdiffusion), whereas the mechanisms and the time-dependencies differ. Envelope solitons are generally more sensitive to damping than pulse solitons. This sensitivity leads to an exponentially growing position variance, which ends when the envelope soliton is destroyed by damping and noise. The pulse solitons have a much longer lifetime and the time-dependence of the position variance proved to have a linear (normal) and a quadratic (superdiffusive) part [21]. The superdiffusion appears to be typical for non-topological solitons because, whereas it was also observed for pulse solitons in classical Heisenberg systems 24, 25], it is absent in the case of topological solitons in the sine-Gordon system [26].

In this article, the aim is to extend the results to chains with long-range interactions (LRI). LRI are necessary extensions which help make the models more realistic. In biomolecules, charged groups or dipole moments (for example the dipole moment of the base pairs in DNA) should have an effect on solitonic or discrete excitations 8, 27, 28]. In the case of the FPU system, it was demonstrated that chains with Kac-Baker or power-law longrange interactions support pulse solitons as well [28, 31]. In the case of Kac-Baker LRI, two different velocity branches for soliton solutions, which are separated by a gap, appear. Low-velocity solutions with a soliton width larger than the interaction radius are pulse-shaped, but develop a cusp when the soliton velocity is close to the critical velocity at the gap [29, 30]. High-velocity solitons with a width in the range of the interaction radius have a multi-component structure, where the center of the soliton is mainly determined by the NNI, whereas the tails are determined by the LRI 31, 32.

According to our research, this is the first known attempt to study the effect of thermal fluctuations on solitons, of wich the shapes are governed by long-range interactions. Our goal is to investigate the consequences of the long-range interactions on the stochastic behavior of the soliton, and specifically on the time-dependence of its position variance.

\section{THE MODEL}

Our model is a one-dimensional chain of equally spaced particles of mass $\mathrm{m}(m=1)$ with interatomic spacing $a$ $(a=1)$. The displacement of the particle $n$ from its equilibrium position is denoted as $u_{n}$. The potential consists of a part $U_{N N}$, with only NNI similar to the original potential chosen by Fermi, Pasta and Ulam, and a longrange part $U_{L R}$ with harmonic coupling $J_{n m}$ between the particles $n$ and $m$ :

$$
L=T-U_{N N}-U_{L R}
$$

with

$$
\begin{array}{r}
T=\frac{1}{2} \sum_{n}\left(\frac{d u_{n}}{d t}\right)^{2}, U_{N N}=\sum_{n} V\left(u_{n+1}-u_{n}\right) \\
U_{L R}=\frac{1}{2} \sum_{n, m} J_{n, m}\left(u_{n}-u_{m}\right)^{2} .
\end{array}
$$

The potential $V(r)$ between nearest neighbors represents the expansion of the Toda potential to third order

$$
V(r)=\frac{1}{2} r^{2}-\frac{1}{3} r^{3}
$$

The long-range coupling between sites $n$ and $m$ is of the Kac-Baker form [33, 34], $\alpha^{-1}$ is the interaction radius of the LRI

$$
J_{n-m}=\frac{1}{2} J\left(e^{\alpha}-1\right) e^{-\alpha|n-m|}, \sum_{m}^{N} J_{m}=J .
$$

For modeling the environment, we choose hydrodynamical damping instead of Stokes damping (because it was demonstrated in 23] that the long-wave components of the Fourier spectrum are strongly damped for Stokes damping which leads to deformations of the soliton) and a Gaussian white noise term which fulfills the fluctuationdissipation-theorem $\left(D^{h y}=2 \nu_{h y} T\right.$ with $k_{B}$ set to unity). The equations of motion in relative displacement coordinates $w_{n}=u_{n+1}-u_{n}$ then take the form

$$
\begin{aligned}
\ddot{w}_{n}= & V^{\prime}\left(w_{n+1}\right)-2 V^{\prime}\left(w_{n}\right)+V^{\prime}\left(w_{n-1}\right) \\
& -\frac{J}{2}\left(e^{\alpha}-1\right) \sum_{m}\left(w_{n}-w_{n+m}\right) e^{-\alpha|m|} \\
& +\nu_{h y}\left(\dot{w}_{n+1}-2 \dot{w}_{n}+\dot{w}_{n-1}\right) \\
& +\sqrt{D^{h y}}\left(\xi_{n+1}(t)-\xi_{n}(t)\right) .
\end{aligned}
$$

In order to apply similar analytic tools as used in [20, 21], we follow Ref. 30 and derive in the quasicontinuum approximation (QCA) a partial differential 
equation (PDE), which is of the Boussinesq (Bq) -type

$$
\begin{gathered}
\partial_{t}^{2} w=\left(c^{2}-1\right) \frac{\kappa^{2} \partial_{x}^{2}}{\kappa^{2}-\partial_{x}^{2}} w+\partial_{x}^{2}\left(w-w^{2}\right)+\lambda \partial_{x}^{2} \partial_{t}^{2} w \\
+\nu_{h y} \partial_{x}^{2} \partial_{t} w+\sqrt{D^{h y}} \partial_{x}^{2} \xi(x, t) \\
\lambda=\frac{1}{12}, \kappa=2 \sinh \left(\frac{\alpha}{2}\right) \\
c=\left[1+\frac{1}{2} J \frac{e^{-\alpha}+1}{\left(e^{-\alpha}-1\right)^{2}}\right]^{\frac{1}{2}}
\end{gathered}
$$

with an additional damping, noise and long-range term (a pseudo differential operator) which is defined as

$$
\left(\alpha^{2}-\partial_{x}^{2}\right)^{-1} f(x)=\frac{1}{2 \alpha} \int_{-\infty}^{\infty} d x^{\prime} e^{-\alpha\left|x-x^{\prime}\right|} f\left(x^{\prime}\right) .
$$

In [30, 31], the solution of equation (6) (for $\nu_{h y}=0$ and $D^{h y}=0$ ) was given implicitly. It was demonstrated that the chain with Kac-Baker LRI supports soliton solutions in the velocity interval $c<v<v_{c}$, where $c$ is the velocity of sound and $v_{c} \simeq \sqrt{\left(4 c^{2}-1\right) / 3}$ is the critical velocity, where the solitons develop a cusp like peakons, which are known from the theory of shallow water waves 37] and the integrable Camassa-Holm equation [38]. A stability analysis in [30] proved that the soliton becomes unstable before it adopts peakon shape. It was further demonstrated that it is possible to construct long-range potentials in nonlinear lattices which support (discrete) peakon solutions 39].

Here, the aim is not to directly solve equation (6), but rather to benefit from the fact that as long as the soliton velocity is smaller than $v_{c}$, the soliton can be relatively well approximated by a pulse-shaped collective variable (CV) ansatz. As demonstrated in Fig. [1] an increasing long-range radius $\alpha^{-1}$ yields larger and broader excitations even when the long-range coupling $J$ is relatively small. For $\alpha=0.5$ and $J=0.1$, the soliton with the normalized velocity $c_{o}=v / c=1.01$ is much broader than the soliton solution without long-range forces $(J=0)$ with the same $c_{o}$. Therefore, the continuum approximation seems to be a very appropriate analytical tool to investigate long-range effects.

The case of nearest neighbor interactions is covered by our theory in the limit $J \rightarrow 0$ (for $\alpha \neq 0$ ). Here, the longrange term in equation ([6) disappears when $\left(c^{2}-1\right) \rightarrow 0$ and we obtain an improved Boussinesq equation (IBq) with $c=1$ 21]. The limit $\alpha \rightarrow \infty$ leads to a IBq with an additional nearest neighbor coupling $J / 2$ and a sound velocity $c=\sqrt{1+J / 2}$.

\section{COLLECTIVE VARIABLES}

A collective variable theory depends crucially on the trial function one chooses to derive ordinary differential equations (ODEs) for the soliton parameters, such
FIG. 1: (Color online) Soliton solutions of eq. (6) with the same normalized velocity $c_{o}=v / c=1.01$ for a chain with nearest neighbor interactions $(J=0)$ and for a chain with a relatively weak long-range coupling $J=0.1$ for different values of the interaction radius $\alpha^{-1}$.

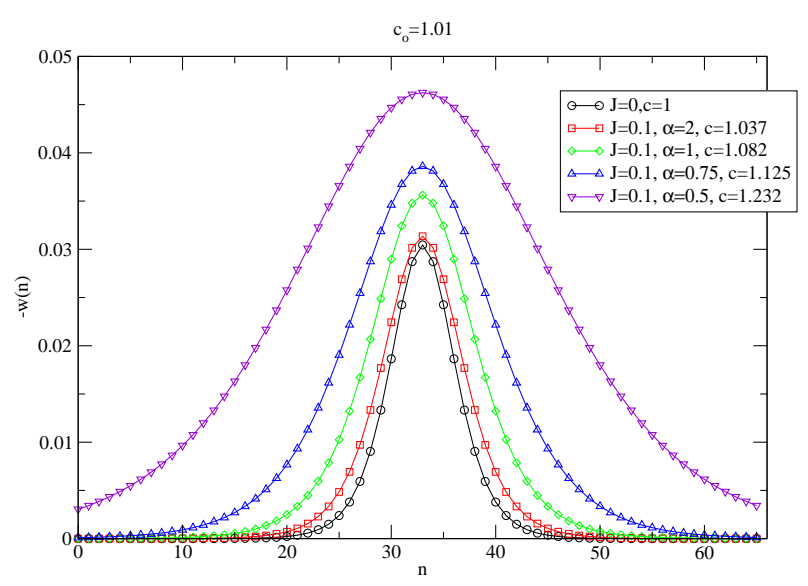

FIG. 2: (Color online) Soliton solution for different values of $c_{o}$ for $J=0.1$ and $\alpha=0.3$. For $c_{o}=v_{c} / c=1.092$, the soliton solution takes the form of a peakon.

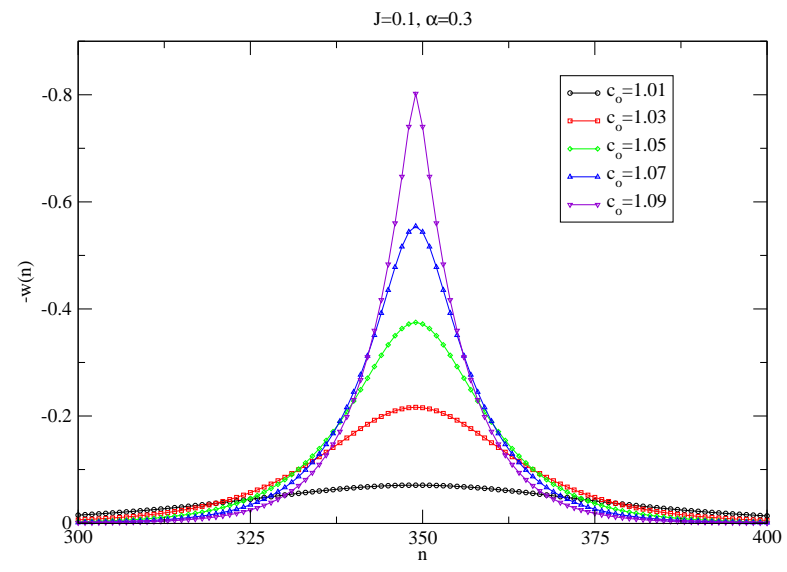

as width and position. As seen in Fig. 2 the shape of the soliton is rather pulse-like up to values $c_{o} \leq 1.07$. A $\mathrm{CV}$ ansatz of this form has already achieved qualitatively good results with simulations for a large range of possible soliton velocities [29]. In absolute displacement coordinates $u(x, t)$, the pulse-shaped soliton solution (in relative displacement coordinates $\left.w(x, t)=\partial_{x} u(x, t)\right)$ takes 
the form of a kink, where the amplitude

$$
2 A_{o}=\int_{-\infty}^{\infty} w(x, t) d x
$$

is a conserved quantity (such as for the Bq equation). Therefore, we choose a trial function of the form

$$
\begin{array}{r}
u(x, t)=A_{o} \tanh (\gamma(t)(x-X(t))) \\
w(x, t)=\partial_{x} u(x, t)=A_{o} \gamma(t) \operatorname{sech}^{2}(\gamma(t)(x-X(t))
\end{array}
$$

with the time-dependent collective coordinates $\gamma(t)$ (inverse width) and $X(t)$ (position) and the kink amplitude $2 A_{o}$ of the unperturbed initial soliton. This $\mathrm{CV}$ ansatz yields the correct results (the Bq soliton solution) in the case of an unperturbed system with only NNI (3)

$$
\begin{array}{r}
\gamma=\sqrt{3} \frac{\sqrt{v^{2}-1}}{v}, A_{o}=\frac{\sqrt{3}}{2} \sqrt{v^{2}-1} v \\
X(t)=v t+X_{o} .
\end{array}
$$

In order to derive ODEs for the collective variables $\gamma(t)$ and $X(t)$, we apply a variational technique similar to the method of Whitham 37]. It is well known that a Lagrange density of Boussinesq-type equations only exists for the absolute coordinate field $u(x, t)$ [4]. In order to include the damping term, the generalized Hamilton principle of Ostrovsky et al. [40, 41], who extended the method of Whitham to nonconservative systems, is here applied. The equation (6) in absolute displacement coordinates

$$
\begin{aligned}
\partial_{t}^{2} u & -\lambda \partial_{x}^{2} \partial_{t}^{2} u-\left(c^{2}-1\right) \frac{\kappa^{2} \partial_{x}^{2}}{\kappa^{2}-\partial_{x}^{2}} u-\partial_{x}^{2} u+2\left(\partial_{x} u\right)\left(\partial_{x}^{2} u\right) \\
& -\sqrt{D^{h y}} \partial_{x} \xi(x, t)=\nu_{h y} \partial_{x}^{2} \partial_{t} u .
\end{aligned}
$$

can be written as

$$
-\frac{\partial \mathcal{L}}{\partial u}+\frac{\partial}{\partial t} \frac{\partial \mathcal{L}}{\partial u_{t}}+\frac{\partial}{\partial x} \frac{\partial \mathcal{L}}{\partial u_{x}}-\frac{\partial^{2}}{\partial x \partial_{t}} \frac{\partial \mathcal{L}}{\partial u_{x t}}=\Phi
$$

with

$$
\begin{array}{r}
\mathcal{L}=\frac{u_{t}^{2}}{2}-\frac{u_{x}^{2}}{2}+\frac{u_{x}^{3}}{3}+\frac{\lambda}{2} u_{x t}^{2}+\sqrt{D^{h y}} u_{x} \xi(x, t) \\
+\frac{\left(c^{2}-1\right) \kappa}{4} \int_{-\infty}^{\infty} e^{-\kappa|x-s|} u(x, t) \partial_{s}^{2} u(s, t) d s \\
\Phi=\nu_{h y} \partial_{x}^{2} \partial_{t} u .
\end{array}
$$

Therefore, the generalized Hamiltonian principle for wave motion in nonlinear and dissipative media in the framework of our CV theory, is used here. Accordingly, the equations of motions can be derived from the averaged Lagrangian density and damping term by varying with respect to the $\mathrm{CV}\left(X_{1}=\gamma(t), X_{2}=X(t)\right)$

$$
\frac{\delta<\mathcal{L}>}{\delta X_{i}}=\frac{\partial<\mathcal{L}>}{\partial X_{i}}-\frac{\partial}{\partial t} \frac{\partial<\mathcal{L}>}{\partial \dot{X}_{i}}=-\left\langle\Phi u_{i}\right\rangle
$$

The brackets signify a spatial integration over $x$. In [20], where the soliton equation was the nonlinear Schrödinger equation, it was possible to include the damping in the Lagrangian density and, thus, no damping term $\Phi$ was needed. Further, the treatment of the damping for the $\mathrm{KdV}$ 21], the treatment of the damping proved unproblematic, because a projection method was used for the CV 42] (this method is, however, rather extensive for the non-local $\mathrm{Bq}$ equation ([6) ).

\section{LANGEVIN EQUATIONS}

Substituting the ansatz (11) in the Lagrangian density (16) and applying the variation (18), results in the following set of stochastic integro-differential equations, where small terms , $\sim \dot{\gamma}^{2}, \sim \ddot{X}$ and $\sim \ddot{\gamma}$ were neglected to achieve analytically manageable equations $(\theta=\kappa / 2 \gamma$, $\bar{x}=x-X(t))$

$$
\begin{aligned}
& \frac{4}{3} \dot{\gamma} \dot{X}-\frac{2 \sqrt{D^{h y}} \gamma^{2}}{A_{o}} \int_{-\infty}^{\infty} \operatorname{Sech}^{2}[\gamma \bar{x}] \operatorname{Tanh}[\gamma \bar{x}] \xi(\bar{x}, t) d \bar{x}= \\
& -\frac{16}{15} \nu_{h y} \gamma^{3} \dot{X} \\
& -2\left(\dot{X}^{2}-1\right)+\frac{16}{9} A_{o} \gamma-4\left(c^{2}-1\right)\left[\theta+\theta^{2}+\theta^{4} \Psi^{\prime \prime}(\theta)\right] \\
& +\frac{2 \sqrt{D^{h y}} \gamma}{A_{o}} \int_{-\infty}^{\infty} d \bar{x} \operatorname{Sech}^{2}[\gamma \bar{x}] \operatorname{Tanh}[\gamma \bar{x}] \bar{x} \xi(\bar{x}, t) \\
& -\frac{2 \sqrt{D^{h y}}}{A_{o}} \int_{-\infty}^{\infty} d \bar{x} \operatorname{Sech}^{2}[\gamma \bar{x}] \xi(x, t)=0 .
\end{aligned}
$$

The values for higher time derivatives of $\gamma(t)$ and $\dot{X}$ can be estimated from the simulation results. A classification of the contributions in orders of a small parameter $\epsilon \sim \gamma_{o}$ makes obvious that these terms contribute only negligibly. It was additionally checked, that the numerical solution of system (20) 43] without noise yields practically the same results as the complete set without neglecting the small terms. At this point, it should be mentioned that the neglect of $\ddot{X}$ is justified by very different arguments compared to those for systems of point particles in the overdamped limit, for instance in ratchet systems. In the following calculations, it will be demonstrated that the dynamics of the system depend on the reduced time scale $t_{r}=\gamma_{o}^{2} \nu_{h y} t$. Therefore, time derivatives appear in an order $\mathcal{O}\left(\epsilon^{4}\right)$ smaller because we choose $\nu_{h y}$ in the or$\operatorname{der} \mathcal{O}\left(\epsilon^{2}\right)$.

The section in the square brackets of (20) results from the long-range interactions, where $\Psi^{\prime \prime}$ is the second derivative of the digamma function. In order to get a first-order equation in $X(t)$, we rewrite equation (20) to

$$
\begin{aligned}
\dot{X}^{2}= & v_{d}^{2}\left(1+\frac{1}{v_{d}^{2}}\left(-\frac{2 \sqrt{D^{h y}}}{A_{o}} \int_{-\infty}^{\infty} d x \operatorname{Sech}^{2}[\gamma \bar{x}] \xi(x, t)+\right.\right. \\
& \left.\left.+\frac{2 \sqrt{D^{h y}} \gamma}{A_{o}} \int_{-\infty}^{\infty} d x \operatorname{Sech}^{2}[\gamma \bar{x}] \operatorname{Tanh}[\gamma \bar{x}] \bar{x} \xi(x, t)\right)\right)
\end{aligned}
$$


with

$$
v_{d}^{2}=1+\frac{8}{9} A_{o} \gamma(t)-2\left(c^{2}-1\right)\left[\theta+\theta^{2}+\theta^{4} \Psi^{\prime \prime}(\theta)\right] \text {. }
$$

For zero temperature, $v_{d}$ is the velocity of the damped soliton where the damping effects enter in the form of the soliton width $\gamma(t)$. To investigate the influence of the noise, the equations for the $\mathrm{CV}$ need to be written as Langevin-type equations. Thus, we approximate $\dot{X}$ in (19) and $v_{d}$ in equation (21) with the initial velocity of the soliton $v$, extract the square root of (21) and approximate the r.h.s. by expanding the root to the first order in the small parameter $\sqrt{D^{h y}}$. The result is a system of stochastic integro-differential equations for the $\mathrm{CV}$ :

$$
\left(\begin{array}{c}
\dot{\gamma} \\
\dot{X}
\end{array}\right)=\left(\begin{array}{c}
A_{1} \\
A_{2}
\end{array}\right)+\int_{-\infty}^{\infty} d x\left(\begin{array}{cc}
B_{11} & 0 \\
B_{21} & B_{22}
\end{array}\right)\left(\begin{array}{l}
\xi \\
\xi
\end{array}\right)
$$

with

$$
\begin{array}{r}
A_{1}=-0.8 \nu_{h y} \gamma^{3}, \quad A_{2}=v_{d} \\
B_{11}=\frac{3}{2} \frac{\sqrt{D^{h y} \gamma^{2}}}{A_{o} v} \operatorname{Sech}^{2}[\gamma \bar{x}] \operatorname{Tanh}[\gamma \bar{x}] \\
B_{21}=-\frac{\sqrt{D^{h y}}}{2 A_{o} v} \operatorname{Sech}^{2}[\gamma \bar{x}] \\
B_{22}=\frac{\sqrt{D^{h y}} \gamma}{2 A_{o} v} \operatorname{Sech}^{2}[\gamma \bar{x}] \operatorname{Tanh}[\gamma \bar{x}] \bar{x} .
\end{array}
$$

As in 20, 21], we proceed to find a statistically equivalent Langevin system (yielding the same Fokker-Planck equation as (23) in the Stratonovich interpretation [44]), which is more convenient for further numerical and analytical studies. The Langevin-system with two independent Gaussian white noise processes reads:

$$
\left(\begin{array}{c}
\dot{\gamma} \\
\dot{X}
\end{array}\right)=\left(\begin{array}{l}
a_{1} \\
a_{2}
\end{array}\right)+\left(\begin{array}{cc}
b_{11} & 0 \\
0 & b_{22}
\end{array}\right)\left(\begin{array}{l}
\xi^{1} \\
\xi^{2}
\end{array}\right)
$$

with

$$
\begin{array}{r}
a_{1}=A_{1}+\frac{1}{4} \frac{D^{h y}}{A_{o}^{2} v^{2}} \gamma^{2}, a_{2}=v_{d} \\
b_{11}=\sqrt{\frac{3}{5} \frac{\sqrt{D^{h y}}}{A_{o} v}} \gamma^{\frac{3}{2}} \\
b_{22}=\sqrt{\left(\frac{1}{6}+\frac{\pi^{2}}{180}\right)} \frac{\sqrt{D^{h y}}}{A_{o} v \gamma^{\frac{1}{2}}}
\end{array}
$$

At this point, it is possible to compare the Langevin system here presented, with the results only for NNI in reference 21]. Despite the facts that the soliton diffusion in 21] was calculated for low-velocity solitons with a $\mathrm{CV}$ ansatz representing a $\mathrm{KdV}$ soliton, and that a different $\mathrm{CV}$ procedure was used, interesting similarities can be observed. The stochastic equation for $\gamma(t)$ in (28) demonstrates, as in [21], a damping induced broadening of the soliton where the drift term is proportional to the damping constant and to the third power of $\gamma\left(\dot{\gamma} \sim \nu_{h y} \gamma^{3}\right)$, plus a small correction which is proportional to $D^{h y}$. The effect of the damping, namely the soliton broadening mechanism, seems to be the same for solitons of the $\mathrm{KdV}$ and the Bq type. However, and more importantly, the long-range interactions do not seem to change this behavior (yet, of course they change the initial condition $\gamma_{o}=\gamma(t=0)$ of the soliton).

In Ref. 21], it is argued that the observed superdiffusive behavior is induced by the stochasticity of $\gamma(t)$ and its effect on the soliton velocity. The fluctuations of the soliton shape lead to fluctuations of the soliton velocity, and therefore, to the superdiffusive terms in the position variance. For envelope solitons, however, it was shown that the superdiffusive mechanism differs. The reason is that the rapid broadening of the envelope due to the damping, and not the changes due to fluctuations, is the main contribution [20].

If it is assumed that, as for envelope solitons [20], the fluctuations of $\gamma(t)$ are negligible and the main contribution to the soliton diffusion results from the noise acting directly on the soliton center $X(t), b_{11}=0$ can be set and we can use the analytical result for the soliton width, which depends on the reduced time $t_{r}=\gamma_{o}^{2} \nu_{h y} t$

$$
\gamma(t)=\frac{\gamma_{o}}{\sqrt{1.6 t_{r}+1}}
$$

to calculate the position variance

$$
\begin{array}{r}
\operatorname{Var}[X]_{b_{11}=0}=\int_{0}^{t} b_{22}^{2}\left(t^{\prime}\right) d t^{\prime} \\
=\left(\frac{1}{6}+\frac{\pi^{2}}{180}\right) \frac{D^{h y}}{A_{o}^{2} v^{2}} \int_{0}^{t} \gamma^{-1}\left(t^{\prime}\right) d t^{\prime} \\
=\frac{k_{B} T}{A_{o}^{2} v^{2}} \frac{5\left(\frac{1}{6}+\frac{\pi^{2}}{180}\right)}{6}\left(\frac{1}{\gamma(t)^{3}}-\frac{1}{\gamma_{o}^{3}}\right) .
\end{array}
$$

Because higher order terms do not appear until $t_{r} \lesssim$ 1 , this result yields an approximately linear timedependence for typical simulation times. Therefore, $\operatorname{Var}[X]_{b_{11}=0}$ will be denoted as the normal diffusion of the soliton below. The result $\operatorname{Var}[X]_{b_{22}=0}$ which we obtain by solving numerically the Langevin system (28) with the constraint $b_{22}=0$ bears only the stochastic contribution of $\gamma(t)$ and can be regarded as the anomalous or superdiffusive part of the soliton position. Although the equality $\operatorname{Var}[X]=\operatorname{Var}[X]_{b_{11}=0}+\operatorname{Var}[X]_{b_{22}=0}$ does not hold, these two quantities can be used to illustrate the weight of normal and superdiffusive effects.

\section{SIMULATIONS}

The time integration of the equations of motion (5) was carried out by use of the Heun method, which is widely used in the solution of partial differential equations or 
difference-differential equations, coupled to either an additive or a multiplicative noise term [45]. Periodic boundary conditions were used to enable the running of long simulation times on systems of typically 2000 particles, clearly larger than the cut-off value for the LRI $(100 / \alpha)$. For a system with LRI, the simulation times increase with the square of the system size $N$. To measure the diffusion of the solitons, over 100 different realizations of the system, which were carried out on a cluster with 48 processors, were averaged.

The results for the position variance $\operatorname{Var}[X(t)]$ measured in the simulations and the numerical solution of the Langevin system (28) for $\alpha=0.3$ (Fig. 3) and $\alpha=0.2$ (Fig. [4) 30] will be compared below. The purely normal and superdiffusive contributions $\operatorname{Var}[X]_{b_{11}=0}$ and $\operatorname{Var}[X]_{b_{22}=0}$ are also displayed to demonstrate that for the shown velocity range $\left(c_{o}=1.03, c_{o}=1.05, c_{o}=\right.$ $1.07)$, the soliton diffusion $\operatorname{Var}[X]$ is practically equal to the superdiffusive part $\operatorname{Var}[X]_{b_{22}=0}$. The long-range coupling $J$ and the inverse long-range radius $\alpha$ are chosen such as to yield a constant value for the sound velocity $c=1.51516$.

The results from both the simulations and Langevin system for $\alpha=0.3$ (Fig. 3) and especially $\alpha=0.2$ (Fig. 44), generally agree well. The normal diffusion of the soliton is negligible for solitons with velocities $c_{o} \geq 1.03$. The smallness of the term $\operatorname{Var}[X(t)]_{b_{11}=0}$ shows that the direct action of the noise on the soliton position $X(t)$ is practically negligible.

The superdiffusive mechanism $\operatorname{Var}[X(t)]_{b_{22}=0}$ describes the soliton diffusion very well and the agreement increasingly betters for larger values of the interaction radius $\alpha^{-1}$. The fluctuations of $\gamma(t)$ yield a fluctuating drift term $v_{d}(\gamma(t))$ and thus a fluctuating $X(t)$ with a stronger than linear variance $\operatorname{Var}[X(t)]_{b_{22}=0}$. Since the agreement for $\alpha=0.2$ (Fig. (4) is even better than for $\alpha=0.3$ (Fig. 3), the influence of the stochastic quantity $\gamma(t)$ on the soliton velocity $v_{d}$ (where the long-range terms contribute) can be regarded as the driving force of the superdiffusive mechanism.

The Kac-Baker form keeps the sum over the coupling constants $J_{m}$ constant (4). Therefore, the almost perfect agreement between $\operatorname{Var}[X]_{b_{22}=0}$, and the simulation results $\operatorname{Var}[X(t)]$ for smaller values of $\alpha$, can be regarded as an effect of the stronger long-range character of the system. The normal diffusion $\operatorname{Var}[X]_{b_{11}=0}$ for the values of $\alpha$ and $c_{o}$ in figures 3 and 4 is only of importance for very small times and yields a clearly smaller slope than the simulation results. This discrepancy appears in 21] for solitons on chains with NNI as well, and it was tackled in 22 by generalizing the results for the soliton diffusion on the Toda lattice to other anharmonic chains. It was demonstrated that the phonon-induced diffusion of the Toda soliton (when it is expressed in terms of the soliton characteristics (velocity, amplitude, width) of solitons on FPU-like chains) can be used to explain the discrepancy for the prediction of the normal diffusion.

In figures 3 and [4 the dominance of the superdiffusion
FIG. 3: Soliton diffusion for different solitons $\left(c_{o}=1.03\right.$, $c_{o}=1.05$ and $\left.c_{o}=1.07\right)$ on a chain with $J=0.1, \alpha=$ $0.3, \nu_{h y}=0.01$ and $T=0.0001$. The results $\operatorname{Var}[X]_{b_{11}=0}$ and $\operatorname{Var}[X]_{b_{22}=0}$ are obtained by solving the system (28) for $b_{11}=0$ and $b_{22}=0$. The result for $\operatorname{Var}[X]_{b_{22}=0}$ illustrates the dominance of the superdiffusive mechanism.

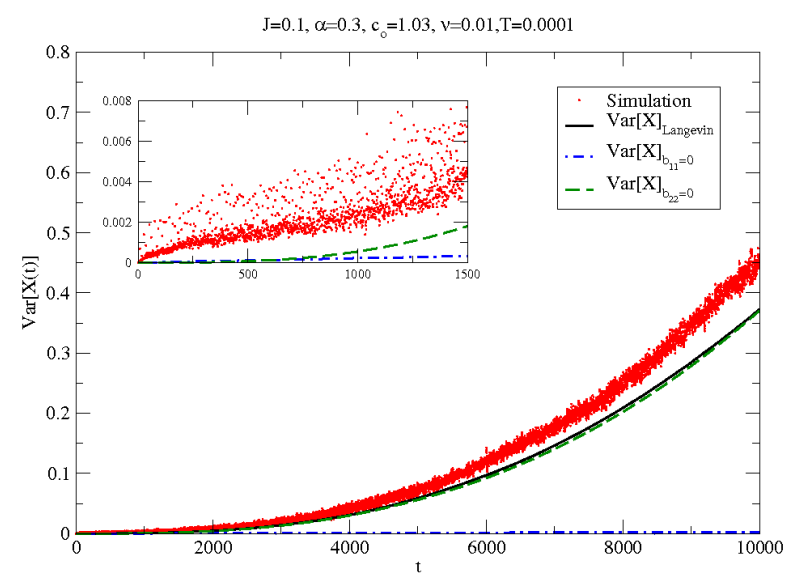

(a)

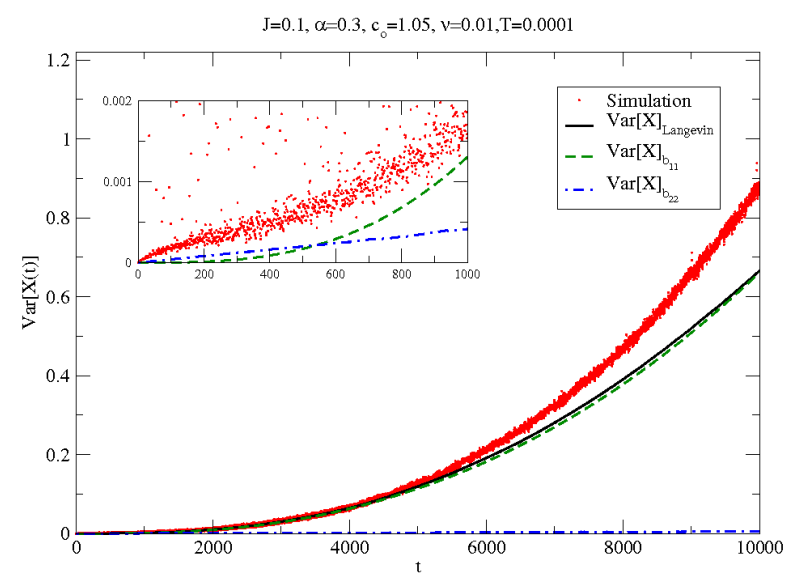

(b)

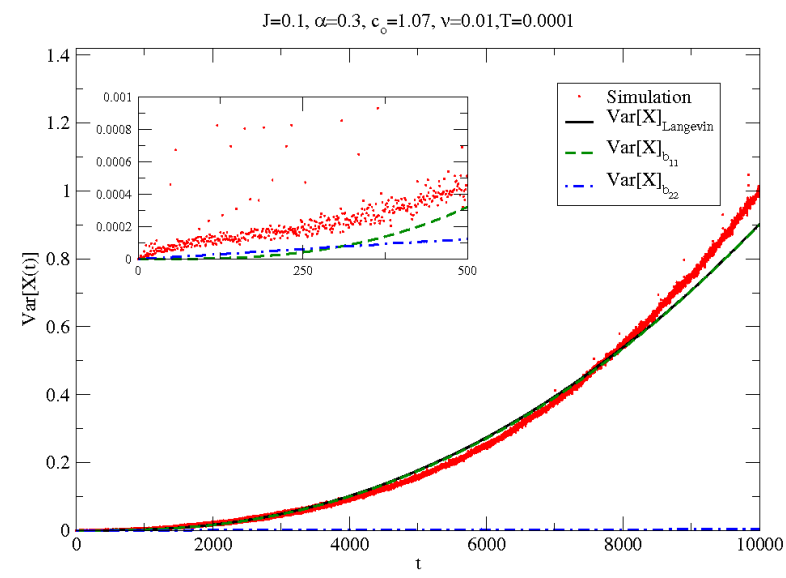

(c) 
FIG. 4: Soliton diffusion for different solitons $\left(c_{o}=1.03\right.$, $c_{o}=1.05$ and $\left.c_{o}=1.07\right)$ on a chain with $J=0.0468, \alpha=$ $0.2, \nu_{h y}=0.01$ and $T=0.0001$. The results $\operatorname{Var}[X]_{b_{11}}=0$ and $\operatorname{Var}[X]_{b_{22}=0}$ are obtained by solving the system (28) for $b_{11}=0$ and $b_{22}=0$. The result for $\operatorname{Var}[X]_{b_{22}=0}$ illustrates the dominance of the superdiffusive mechanism. The small off-set in (a) results from the inaccuracy of measuring the soliton position for very broad solitons.

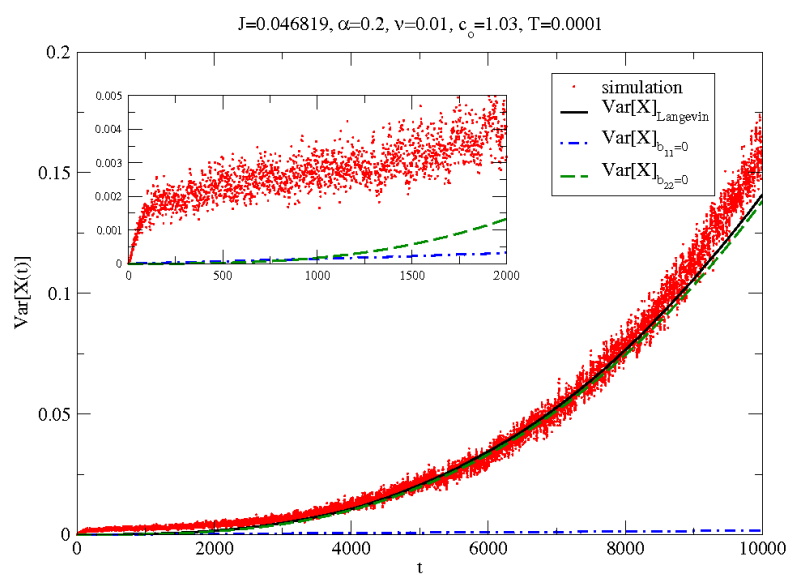

(a)

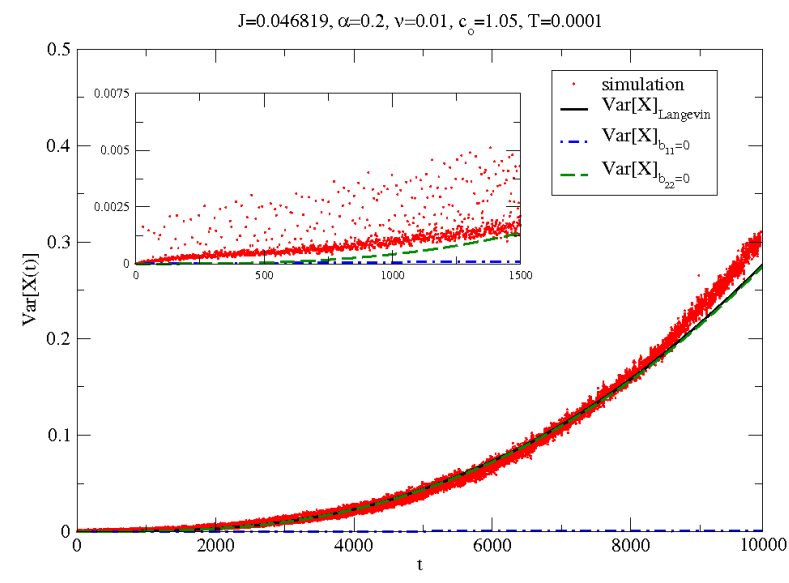

(b)

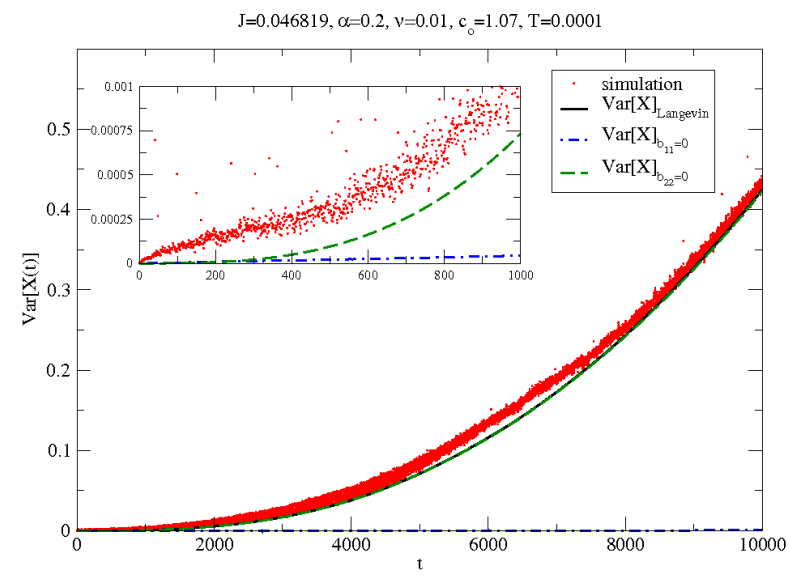

sets in earlier for larger values of $c_{o}$. This result can be qualitatively explained by the fact that the soliton dynamics depends on the time scale $t_{r}$ (30), which is proportional to the damping and the square of the inverse width. For large values of $c_{o}$, the width is small and the reduced time $t_{r}$ increases faster. Interestingly enough, the time coordinate $\tau$ in the analytical result for the position variance of the $\mathrm{KdV}$ solitons 21] appears with a pre-factor $\lambda$ which is also proportional to the damping and the square of the inverse width. The dependence of the soliton diffusion on such a time scale seems very typical and is ultimately a consequence of the universal behavior of the soliton width in the presence of damping (30).

To quantitatively see the influence of the long-range radius $\alpha^{-1}$ and the time scale $t_{r}$ on the soliton diffusion, an analytical result for the position variance would have to be deduced. However, because the dependence of $v_{d}$ on $\theta$ involves a polygamma function (22), this is a rather hard task. According to our observation, the superdiffusive part of the simulation results can always be very well approximated by a mainly quadratic and cubic time dependence. This observation agrees with the result of a perturbation theory in 21] that the superdiffusion yields a term $\sim t^{2}$ for the position variance of the $\mathrm{KdV}$ soliton. For solitons with relatively low velocities, the normal diffusion is more pronounced than for their counterparts on NNI chains for simulation times $t<10000$. The LRI solitons become very broad for small velocities and appear more as a collective excitation of the system, than localized energy pulses (see Fig 1 for $c_{o}=1.01$ ). The superdiffusive character for solitons, with for example $c_{o}=1.01$, appears only for $t>10000$, when $t_{r}$ reaches larger values.

The theory of section IV holds for chains with only nearest neighbor interactions (by setting $J=0$ ) as well. In this case, the results agree well to those of earlier studies 21, 46]. The diffusive mechanism is basically the same as for LRI, but because the discreteness effects are much stronger, the solitons for the nearest neighbor case are rather narrow and can not manage velocities as high as for LRI. Therefore, the normal diffusion is dominant for broad solitons at quite small soliton velocities, for example $c_{o}=1.005$. Superdiffusive effects can hardly be seen, because these low-energy solitons are rapidly destroyed by the noise and damping. For larger values of $c_{o}$, such as $c_{o}=1.01$ and $c_{o}=1.02$ (in Figures [5] a and b), the superdiffusive contribution dominates.

The numerical results for the soliton diffusion on chains with Kac-Baker long-range interactions generally suggest that the superdiffusive mechanism is more pronounced than it is for nearest neighbor interactions. For LRI, the range of possible velocities where the superdiffusion dominates is clearly enlarged. The solitons in the LRI case also have higher energies and longer life times which because of the quadratic and cubic terms in the timedependence of $\operatorname{Var}[X(t)]$, lead to the dominance of the superdiffusion. 
FIG. 5: Soliton diffusion for different soliton velocities $\left(c_{o}=\right.$ 1.01 and $\left.c_{o}=1.02\right)$ on a chain with only nearest neighbor interactions $(J=0, c=1)$ and $\nu_{h y}=0.003$ and $T=5 \cdot 10^{-6}$.

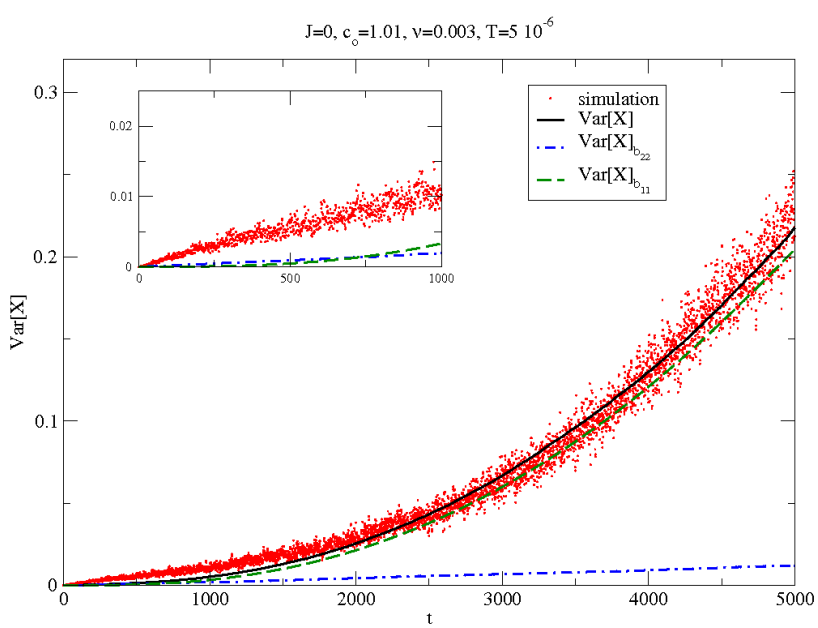

(a)

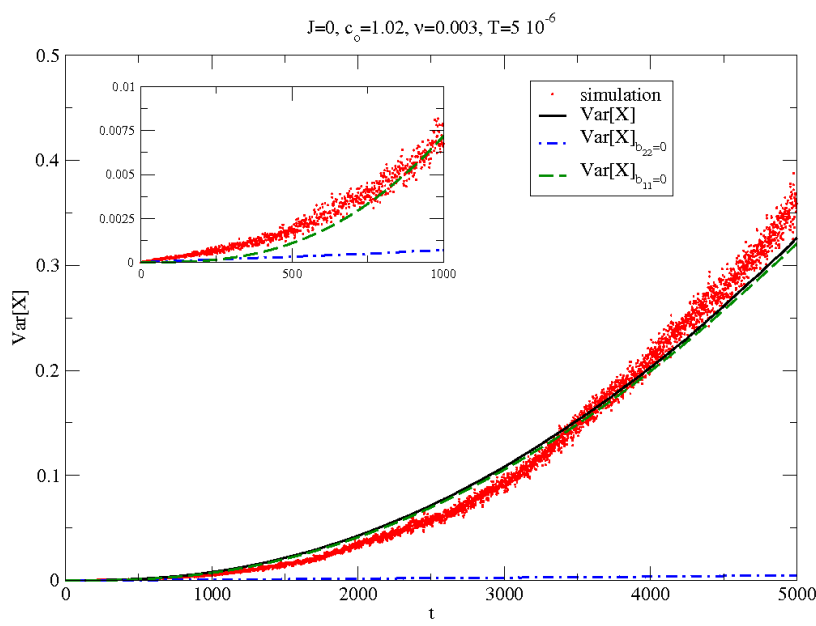

(b)

\section{SMALL-NOISE EXPANSION AND LONG-TIME LIMIT}

In order to gain some deeper insight in the diffusion mechanism of the long-range solitons and the timedependece of $\operatorname{Var}[X(t)]$, we develop a small-noise expansion of the Langevin set (28). The most important step in the analytical treatment is the rather complicated dependence of the velocity $v_{d}$ on the inverse width $\gamma(t)$. An expansion of the polygamma function $\Psi^{\prime \prime}(\theta) \approx-\frac{1}{\theta^{2}}-\frac{1}{\theta^{3}}-\frac{1}{2 \theta^{4}}+\frac{1}{6 \theta^{6}}$ for large $\theta=\kappa / 2 \gamma$ [50] is already rather appropriate for typical values of $\theta(t=0) \in[1.5,3]$ for the considered long-range solitons with $c=1.51516$ in Fig. 3] and Fig. 4] This approximation is even for smaller values of $\theta$ justified after some time $t>1 / \gamma^{2} \nu_{h y}$, because of the damping-induced growing of the soliton width $\gamma^{-1}$. It can be shown that the soliton velocity $v_{d}$ depends in this approximation only on an additional term which is proportional to $\gamma^{2}$ :

$$
\begin{aligned}
v_{d} & =\sqrt{1+\frac{8}{9} A_{o} \gamma(t)-2\left(c^{2}-1\right)\left[\theta+\theta^{2}+\theta^{4} \Psi^{\prime \prime}(\theta)\right]} \\
& \approx \sqrt{1+\frac{8}{9} A_{o} \gamma(t)-2\left(c^{2}-1\right)\left[-\frac{1}{2}+\frac{1}{6 \theta^{2}}\right]} \\
& \approx c \sqrt{1+\frac{8}{9} \frac{A_{o}}{c^{2}} \gamma(t)-\frac{4}{3} \frac{\left(c^{2}-1\right)}{c^{2} \kappa^{2}} \gamma^{2}} \\
& \approx c+\frac{4}{9} \frac{A_{o}}{c} \gamma(t)-\left(\frac{8}{81} \frac{A_{o}^{2}}{c^{3}}+\frac{2}{3} \frac{\left(c^{2}-1\right)}{c \kappa^{2}}\right) \gamma(t)^{2} .
\end{aligned}
$$

The small-noise expansion of the Langevin set (28) follows reference [51] and was already successfully applied to the problem of the diffusion of low-velocity solitons in the FPU system without long-range interactions [21]. We seek an asymptotic solution of the form

$$
\begin{array}{r}
\gamma(t)=\gamma^{(0)}(t)+\epsilon \gamma^{(1)}(t)+\ldots \\
X(t)=X^{(0)}(t)+\epsilon X^{(1)}(t)+\ldots
\end{array}
$$

where $\epsilon$ is a small parameter which is formally introduced to consider the influence of the noise terms as small perturbations $\left(\sqrt{D^{h y}} \sim \epsilon\right)$. The contribution of the drift term in the different orders of $\epsilon$ are calculated following the rule

$$
\begin{aligned}
a_{i}(\gamma(t)) & =a_{i}\left(\gamma^{(0)}+\sum_{m=1}^{\infty} \epsilon^{m} \gamma^{(m)}(t)\right) \\
& =a_{i}^{(0)}(t)+\epsilon \gamma^{(1)}(t) \frac{d a_{i}\left(\gamma^{(0)}(t)\right)}{d \gamma^{(0)}(t)}+\ldots
\end{aligned}
$$

With $a_{2}=v_{d}$, the equations in the order $\epsilon^{0}$ read:

$$
\begin{aligned}
d \gamma^{(0)}(t) & =-0.8 \nu_{h y} \gamma^{(0)}(t)^{3} d t \\
d X^{(0)}(t) & =\left(c+\frac{4}{9} \frac{A_{o}}{c} \gamma(t)\right. \\
& \left.-\left(\frac{8}{81} \frac{A_{o}^{2}}{c^{3}}+\frac{2}{3} \frac{\left(c^{2}-1\right)}{c \kappa^{2}}\right) \gamma(t)^{2}\right) d t
\end{aligned}
$$

and describe the damped soliton. The first corrections due to the noise terms appear in the next order $\epsilon^{1}$ :

$$
\begin{aligned}
d \gamma^{(1)}(t) & =-2.4 \nu_{h y} \gamma^{(1)}(t) \gamma^{(0)}(t)^{2} d t \\
& +\sqrt{\frac{3}{5}} \frac{\sqrt{D} \gamma^{(0)}(t)^{\frac{3}{2}}}{A_{o} v} d W_{1} \\
d X^{(1)}(t) & =\gamma^{(1)}(t)\left(c+\frac{4}{9} \frac{A_{o}}{c}\right. \\
& \left.-\left(\frac{16}{81} \frac{A_{o}^{2}}{c^{3}}+\frac{4}{3} \frac{\left(c^{2}-1\right)}{c \kappa^{2}}\right) \gamma^{(0)}(t)\right) d t \\
& +\sqrt{\frac{1}{6}+\frac{\pi^{2}}{180}} \frac{\sqrt{D}}{\sqrt{\gamma^{(0)}} A_{o} v} d W_{2} .
\end{aligned}
$$


If we substitute the result for $\gamma^{(1)}$ and $\gamma^{(0)}=$ $\gamma_{o} / \sqrt{1.6 \nu_{h y} \gamma_{o}^{2} t+1}$ into (39), we can calculate the firstorder expression for the variance of the soliton position due to the noise

$$
\operatorname{Var}\left[X_{1}(t)\right]=\lim _{s \rightarrow t}<X_{1}(t) X_{1}(s)>.
$$

This is the first order result for the soliton position variance $\operatorname{Var}[X(t)]$ when we finally set $\epsilon=1$ which is justified if the noise in the system is sufficiently small. Since the small-noise expansion yields a very lengthy result for the position variance, which depends on the time scale $t_{r}=1.6 \nu_{h y} \gamma_{o}^{2} t$, we prefer to demonstrate the good agreement just for one typical example (Fig. 6).

FIG. 6: Simulation results for the position variance of a soliton with $c_{o}=1.03$ on a chain with $J=0.046819, \alpha=0.2$, $c=1.51516, \nu_{h y}=0.01$ and $T=0.0001$. The results agree well with the numerical solution of the Langevin system (28). The result from the small-noise expansion reproduces the numerical solution of the Langevin system qualitatively well.

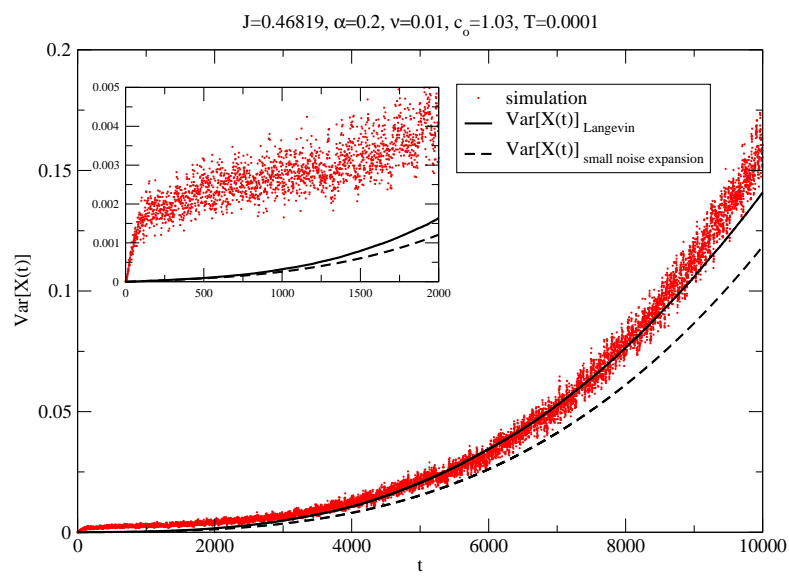

(a)

Further comparisons reveal that the analytical result for $\operatorname{Var}[X(t)]$ begins to fail for high velocities $v$ close to the critical velocity $v_{c}$. The reason is the restriction to the first order corrections. For high-velocity solitons, the width is quite narrow and the fluctuations of the width are strong and can no longer be well described by $\gamma^{(1)}(t)$. The long-time limit for the soliton diffusion result in the small-noise expansion predicts an asymtotic soliton diffusion where the underlying soliton shape determines only the numerical constant in

$$
\operatorname{Var}[X(t)]_{\text {inf }}=\frac{1}{64} \frac{D^{h y}}{c^{2} v^{2} \nu_{h y}^{1.5}} t^{3 / 2} .
$$

We can not prove this prediction in our simulations because the result $\operatorname{Var}[\mathrm{X}(\mathrm{t})]$ for the small-noise expansion reaches the asymptotic $t^{3 / 2}$ time-dependence after very long times. It would take times larger than $10^{6}$ for the soliton of Fig. 6] For these times, the soliton is not a localized energy pulse but smeared out over the system and the soliton velocity $v$ approaches the velocity of sound $c$. Therefore, we do not assume the long-time limit to play a role in energy transfer mechanisms. As long as the soliton is not too broad, the time dependence of the soliton diffusion can be well approximated by a quadratic and cubic term in $t$.

\section{CONCLUSIONS}

It was observed that the diffusion of solitons on chains with Kac-Baker long-range interactions is in many respects very similar to their counterparts on chains with nearest neighbour interactions. A theory which describes both cases very well, was derived here. Especially the dependence of $\gamma(t)$ on $t_{r}$, and the superdiffusive mechanism with the mainly quadratic contribution in time to the position variance, in both cases, gives rise to the conjecture that superdiffusion is a generic feature for nontopological solitons.

In the case of long-range interactions, the solitons can adopt rather high velocities and it was observed that the superdiffusive mechanism of the Langevin system, which was derived in the framework of the applied CV theory for the non-local Boussinesq equation with noise and damping terms, describe them well. The prediction of the derived Langevin set for the superdiffusion of the soliton is especially accurate for small values of $\alpha$, where solitons (for a certain velocity $c_{o}$ ) possess more energy than for larger values of $\alpha$. It is not surprising that the superdiffusion dominates for massive solitons because the direct influence of the noise on the soliton position is less important. The superdiffusion is more present for solitons with LRI, because the possible velocity range and the lifetime of the solitons are distinctly enlarged. For a chain with nearest neighbor coupling, solitons could only be described to rather moderate velocities, where the normal diffusion is more present.

In the framework of a small-noise expansion for the Langevin system (28), we derived an analytical result for the position variance $\operatorname{Var}[X(t)]$ which agreed well with the simulations when the soliton velocities are not too large. This result depends in a quite complicated way on the time scale $t_{r}$. But the result confirms the former observation that the superdiffusion yields contributions in $t^{2}$ which turn into a $t^{3 / 2}$ dependence for long times when the soliton is rather broad with a velocity $v$ close to the sound velocity $c$.

For small times, the soliton with LRI showed a normal diffusion which is larger than the result $\operatorname{Var}[X(t)]_{b_{11}=0}$ of the CV theory. The problem in determining the normal diffusion of the soliton may be two-fold. In the case of a chain with nearest neighbor coupling [21, 22], it was established that the phonons have a significant contribution to the diffusion of solitons, especially for low-velocity solitons. This result could possibly be similar for LRI 
solitons. When, as in this paper, higher velocities are examined, the $\mathrm{KdV}$ equation is no longer suitable describing the situation well. Instead, the Bq equation, where a second-order time derivative of the wave function appears, which results in ODEs for the CVs where the soliton position appears quadratic $\left(\dot{X}^{2}\right)$, has to be used. To approximate this result by a Langevin-type equation, the square root of the r.h.s. of (21) had to be expanded because this procedure yields the correct velocity $v_{d}$ without noise. However, with this procedure and by substituting $v_{d}$ with a constant value $v$, the result has the tendency to underestimate the influence of the noise on the CV $X$. Thus, it is difficult to decide wether it is the phonons or the applied approximations, which are responsible for the observed discrepancies.

Nevertheless, the predicted superdiffusive behavior of localized energy pulses in systems with long-range forces can be observed for long enough times. Moreover, the long-range interactions lead to much larger soliton energies and lifetimes, so that the soliton diffusion could be an observable effect in real systems.
[1] A.C. Scott, Nonlinear Science, University Press Oxford (1999)

[2] AC Scott (editor), The Encyclopedia of Nonlinear Science, Routledge, New York, 2005

[3] E. Fermi, J. Pasta, and S. Ulam, Collected Works of Enrico Fermi (University of Chicago, Chicago, 1965), Vol. II, p. 978 .

[4] D.K. Campbell, P. Rosenau and G.M. Zaslavsky, Chaos 15, 015101 (2005)

[5] M. Peyrard, Nonlinearity 17, R1 (2004)

[6] L. V. Yakushevich, J. Biosci., 26, 305 (2001)

[7] T. Dauxois, M. Peyrard, Physics of Solitons, Cambridge University Press (2005)

[8] S.F. Mingaleev, P.L. Christiansen, Yu.B. Gaididei, M. Johansson and K.Ø. Rasmussen, J. of Biological Physics 25, 41 (1999)

[9] C.H. Choi, G. Kalosakas, K.Ø. Rasmussen, M. Hiromura, A. R. Bishop and A. Usheva, Nucleic Acids Res. 32, 1584 (2004)

[10] A.C. Scott, Physics Reports, 217, 1-67 (1992)

[11] A.S. Davydov, Solitons in Molecular Systems, (Reidel, Dotrecht, 1985)

[12] J. Edler, P. Hamm, A.C. Scott, Phys. Rev. Let. 88, 067403 (2002)

[13] S. Yomosa, Phys. Rev. A 32, 1752 (1985)

[14] P. Perez, N. Theodorakopoulos, Phys. Lett. A 117, 405 (1986)

[15] P. Perez, N. Theodorakopoulos, Phys. Lett. A 124, 267 (1987)

[16] D. Hochstrasser, F.G. Mertens, and H. Büttner, Phys. Rev. A 40, 2602

[17] P.L. Christiansen, A.V. Zolotaryuk, and A.V. Savin, Phys. Rev. E 56 (1997)

[18] F. d'Ovidio, H.G. Bohr, and P. Lindgård, J. Phys.: Condens. Matter 15, s1699 (2003)

[19] F. d'Ovidio, H.G. Bohr, and P. Lindgård, Phys. Rev. E 71, 026606 (2005)

[20] C. Brunhuber, F.G. Mertens, Y. Gaididei, Eur. Phys. J. B 42, 103 (2004)

[21] E. Arévalo, F. G. Mertens, Y. Gaididei, A. R. Bishop, Phys. Rev. E 67, 016610 (2003)

[22] F. G. Mertens, E. Arévalo, and A.R. Bishop, Phys. Rev. E 72, 036617 (2005)

[23] E. Arévalo, F. G. Mertens, and A.R. Bishop, Eur. Phys. J. B 2763 (2002)

[24] T. Kamppeter, F.G. Mertens, E. Moro, A. Sánchez, A.R. Bishop, Phys. Rev. B 59, 11349 (1999)
[25] M. Meister, F.G. Mertens, Y. Gaididei, A.R. Bishop, Eur. Phys. J. B 20, 405 (2001)

[26] N. Quintero, A. Sánchez, F.G. Mertens, Eur. Phys. J.. B 16, 361 (2000)

[27] JFR Archilla, Y.B. Gaididei, P.L. Christiansen, and J. Cuevas, J. Phys. A: Math. Gen. 35, 8885 (2002)

[28] S.F. Mingaleev, Y.B. Gaididei, and F.G. Mertens, Phys. Rev. E 58, 3833 (1998)

[29] A. Neuper, Y.B. Gaididei, N. Flytzanis, and F.G. Mertens, Phys. Lett. A 190, 165

[30] Y.B. Gaididei, N. Flytzanis, A. Neuper, and F.G. Mertens, Physica D 107, 83

[31] S.F. Mingaleev, Y.B. Gaididei, and F.G. Mertens, Phys. Rev. E 61, R1044

[32] P.L. Christiansen, Y.B. Gaididei, F.G. Mertens and S.F. Mingaleev, Eur. Phys. J. B 19, 545

[33] G.A. Baker Jr, Phys. Rev. 122, 1477 (1961)

[34] A.M. Kac and B.C. Helfand, J. Math. Phys. 4, 1078 (1972)

[35] P.L. Christiansen, Y.B. Gaididei, F.G. Mertens and S.F. Mingaleev, Eur. Phys. J. B 19, 545

[36] Dauxois, Ruffo, Arimondo, Wilkens (Eds). Dynamics and Thermodynamics of Systems with Long-Range Interactions, Lecture Notes in Physics 602, Springer (2002)

[37] G.B. Whitham, Linear and Nonlinear Waves (Wiley,New York, 1974)

[38] R. Camassa and D.D. Holm, Phys. Rev. Lett. 71, 1661

[39] A. Comech, J. Cuevas, and P.G. Kevrekidis, Physica D 207, 137 (2005)

[40] L.A. Ostrovsky and E.N. Pelinovsky, Sov. Phys. Dokl. 15, 1097 (1971)

[41] A. Jeffrey and T.Kawahara, Asymptotic Methods in Nonlinear Wave Theory, Pitman, London, 1982

[42] F.G. Mertens, H.J. Schnitzer, and A.R. Bishop, Phys. Rev. B 56, 2510 (1997)

[43] S. Wolfram, The Mathematica Book (Wolfram Media, Cambridge University Press, Cambridge, 1996).

[44] V. Konotop, L. Vázquez, Nonlinear Random Waves (World Scientific, Singapore, 1994)

[45] P. Kloeden, E. Platen, Numerical solution of Stochastic Differential Equations, Springer-Verlag, New YorkHeidelberg-Berlin, 1994

[46] E. Arévalo, Dissertation, Universitaet Bayreuth (2004)

[47] N. J. Zabusky, M. D. Kruskal, Phys. Rev. Lett. 15, 240 (1965)

[48] N. Flytzanis, S. Pnevmatikos, M. Remoissenet, J. Phys. $\mathrm{C}$ 
[49] T. Dauxois, M. Peyrard, A. R. Bishop, Phys. Rev. E 47, 684 (1993)

[50] M. Abramowitz and I. Stegun, Handbook of Mathematical Functions with Formulas, Graphs and Mathematical Tables. Dover Publications, Inc., New York, Ninth Printing

\section{November 1970}

[51] C.W. Gardiner,Handbook of Stochastic Methods, Springer-Verlag, New York-Heidelberg-Berlin, 1983 